\title{
Transverse domain walls in nanoconstrictions
}

\author{
D. Backes, ${ }^{\text {a) }}$ C. Schieback, M. Kläui, ${ }^{\text {b) }}$ F. Junginger, H. Ehrke, P. Nielaba, and U. Rüdiger \\ Fachbereich Physik, Universität Konstanz, Universitätsstrasse 10, 78457 Konstanz, Germany \\ L. J. Heyderman \\ Laboratory for Micro- and Nanotechnology, Paul Scherrer Institut, 5232 Villigen PSI, Switzerland
}

C. S. Chen, T. Kasama, and R. E. Dunin-Borkowski

Department of Materials Science and Metallurgy, University of Cambridge, Pembroke Street, Cambridge CB2 3QZ, United Kingdom

C. A. F. Vaz ${ }^{\mathrm{c})}$ and J. A. C. Bland

Cavendish Laboratory, University of Cambridge, Madingley Road, Cambridge CB3 OHE, United Kingdom

(Received 19 June 2007; accepted 13 August 2007; published online 11 September 2007)

\begin{abstract}
The spin structure of domain walls in constrictions down to $30 \mathrm{~nm}$ is investigated both experimentally with electron holography and with simulations using a Heisenberg model. Symmetric and asymmetric transverse domain walls for different constriction sizes are observed, consistent with simulations. The experimentally observed asymmetric transverse walls can be further divided into tilted and buckled walls, the latter being an intermediate state just before the vortex nucleation. As the constriction width decreases, the domain wall width decreases faster than linearly, which leads to very narrow domain walls for narrow constrictions. (C) 2007 American Institute of Physics. [DOI: 10.1063/1.2779109]
\end{abstract}

Control and manipulation of magnetic domain walls (DWs) are in the focus of interest because of the associated exciting physical phenomena and the potential for applications such as magnetic logic and data storage devices. ${ }^{1,2}$ Here, reproducible and controlled switching by DW motion, induced either by an external magnetic field or a spinpolarized current, is essential. ${ }^{3-5}$ The detailed DW spin structure and width play a very important role in determining the DW velocity in current-induced ${ }^{5}$ and field-induced motion. ${ }^{6,7}$ For very narrow walls, nonadiabatic contributions to the electron transport are predicted to become significant, ${ }^{8-10}$ which would increase the current-induced DW velocity, which is important for applications. Reciprocal effects of the spin structure on the magnetotransport, such as DW magnetoresistance, also depend on the detailed wall spin structure. $^{11,12}$

On reducing the lateral dimensions, it is the geometry rather than the material parameters which determines the DW type and spin structure. ${ }^{13-16}$ For a one dimensional chain model, it has been predicted that the reduction of the lateral dimensions leads to a reduction in the Bloch DW width. ${ }^{13}$ For $180^{\circ}$ Néel walls in Permalloy $\left(\mathrm{Fe}_{20} \mathrm{Ni}_{80}\right)$ thin film rectangular structures, the reduction of the DW width has been studied using scanning electron microscopy with polarization analysis with a magnetic resolution of $20-30 \mathrm{~nm} .{ }^{17}$ The types of DWs confined in wires or ring elements are very different from these Néel walls, since they exhibit head-tohead wall structures with two types prevailing: transverse and vortex DWs. ${ }^{14,15}$ Using transmission electron microscopy techniques, the spin structure of head-to-head DWs in

\footnotetext{
a) Also at: Laboratory for Micro- and Nanotechnology, Paul Scherrer Institut, 5232 Villigen PSI, Switzerland.

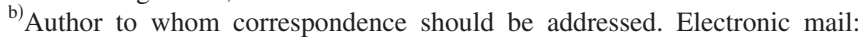
Mathias.Klaeui@uni-konstanz.de

${ }^{c}$ Present address: Applied Physics, Yale University, New Haven, Connecticut 06520 .
}

elements down to $200 \mathrm{~nm}$ lateral size has been measured. ${ }^{18}$ Observations of DWs in constrictions have also been carried out to determine the pinning potential, but the detailed spin structures and wall widths have not been ascertained. ${ }^{19}$

Employing micromagnetic simulations, the geometry dependence of the wall types (wall phase diagram) has been studied down to $20 \mathrm{~nm}$ width. In addition to the transverse and vortex walls, asymmetric transverse walls were predicted. ${ }^{16}$ In the conventional micromagnetic approach used in Ref. 16, the exchange energy is approximated by $(\vec{\nabla} \cdot \vec{m})^{2}(\vec{m}$ is the local magnetization), which is the first order Taylor expansion of the dot product and only valid for small angles between neighboring cells. ${ }^{20,21}$ However, geometrically confined DWs contain changes in the spin structure at very short length scales. ${ }^{13}$ In order to address this problem, an atomistic/semiclassical spin model approach can be used, where the exchange energy is calculated as the dot product. ${ }^{22}$

In this letter, we investigate experimentally with electron holography and with numerical simulations the spin structure of DWs in constrictions down to $30 \mathrm{~nm}$. We find three DW types which differ in their spin configuration and we characterize them by their DW opening angle. From this an average DW width is calculated and the dependence on the constriction width is determined. We compare the measured DWs with the results of computer simulations using an extended Heisenberg model.

We fabricated constrictions down to $30 \mathrm{~nm}$ by introducing triangular notches in curved line magnetic elements. A schematic of such magnetic elements with element width $w_{e}$ and constriction width $w_{c}$ is shown in Fig. 1(a). The elements were fabricated using electron beam lithography on silicon nitride $\left(\mathrm{Si}_{3} \mathrm{~N}_{4}\right)$ membrane substrates required for off-axis electron holography observations, as described in Ref. 23. This technique, with its sub-10 nm resolution allows for the observation of the spin structure in the smallest constrictions. Here, the magnitude and the direction of the magnetic induc- 


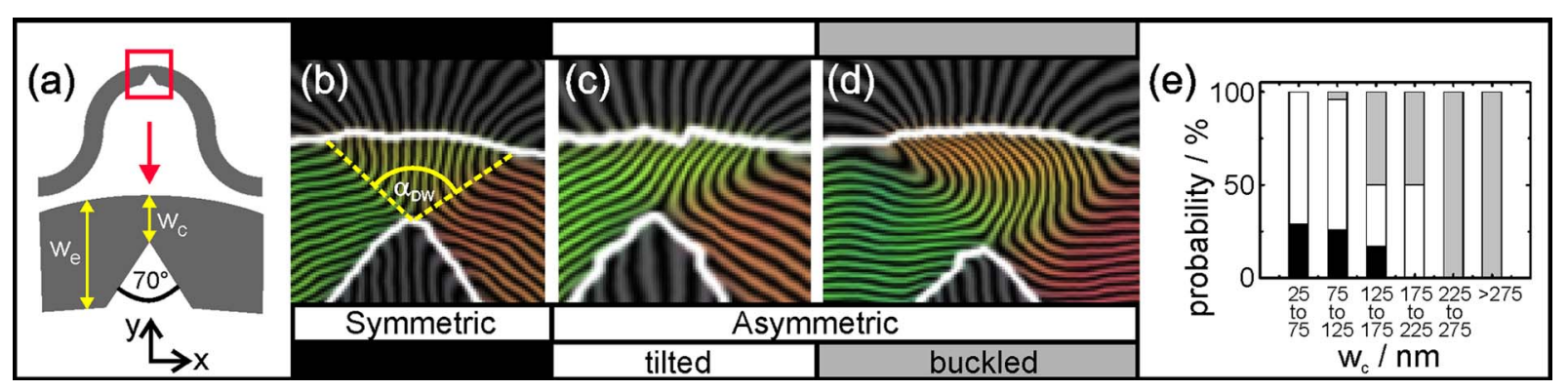

FIG. 1. (Color online) (a) Schematic of the Permalloy element geometry, with element width $w_{e}$, constriction width $w_{c}$, and notch angle of $70^{\circ}$. The DW opening angle $\alpha_{\mathrm{DW}}$ is shown in (b). [(b)-(d)] Off-axis electron holography images of the observed transverse DW types with a thickness of $11 \mathrm{~nm}$ and $w_{c} / w_{e}$ : (b) $138 \mathrm{~nm} / 400 \mathrm{~nm}$, (c) $103 \mathrm{~nm} / 300 \mathrm{~nm}$, and (d) $191 \mathrm{~nm} / 300 \mathrm{~nm}$. The color code for the magnetization direction is given in Fig. 3. (e) Distribution in given ranges of $w_{c}$ of transverse DW types: symmetric (black), asymmetric tilted (white), and asymmetric buckled (gray).

tion in the element are determined from the phase shift of an electron wave when transmitted through a magnetic element. An electron biprism splits the electron beam into two parts; one passing through the element and the other passing through the membrane as a reference wave. The phase information is extracted from the interference pattern, and from this a magnetic induction map is obtained. ${ }^{24}$ The magnetic elements consist of Permalloy films $\left(w_{e}=100-400 \mathrm{~nm}\right)$, deposited in an ultrahigh vacuum molecular beam epitaxy deposition chamber, with thicknesses below $20 \mathrm{~nm}$ in order to obtain transverse DWs. ${ }^{15}$ The DWs could be nucleated using the magnetic field produced by the objective lens of the detection microscope.

From the high spatial resolution electron holography images, we were able to determine systematically the shape of the DWs even in the smallest constrictions. While it is already known that transverse walls are located within the constriction and vortex walls are located adjacent to it depending on geometry, ${ }^{19}$ we concentrate on the detailed spin structure of transverse walls in constrictions varying $w_{c}, w_{e}$, and the element's thickness. In agreement with micromagnetic prediction of symmetric and asymmetric walls, ${ }^{16}$ we also experimentally find symmetric transverse walls [Fig. 1(b)], asymmetric tilted [Fig. 1(c)], and asymmetric buckled transverse DWs [Fig. 1(d)]. Figure 1(e) shows the percentage of each wall type in different constriction ranges. In line with the results in Ref. 16, we find symmetric walls only in narrow constrictions and asymmetric walls prevailing for wider constrictions. Both tilted asymmetric and symmetric walls appear in the range of $w_{c}<175 \mathrm{~nm}$. The second asymmetric wall type has a buckled shape [Fig. 1(d)] and can be found for large constrictions, $w_{c}=75-370 \mathrm{~nm}$. Since in the thick samples with large constrictions, vortex walls become the most favorable DW type, we can consider buckled DWs as an intermediate state at the onset of the transformation from a transverse wall to a vortex wall where the vortex core is not yet nucleated.

As seen in Figs. 1(b)-1(d), the magnetization configuration varies significantly moving in the positive $y$ direction [see Fig. 1(a)] from the tip of the notch toward the outside edge of the element, and it is not obvious how the DW width should be defined. Traditionally, the widths of $180^{\circ}$ Bloch walls have been defined as $\lambda$ from the $\tanh (x / \lambda)$ magnetization profile ${ }^{25}$ and this definition is commonly applied to $180^{\circ}$ Néel walls. Due to the more complicated spin structure of the head-to-head walls, this is not applicable in our case. The profiles of head-to-head walls exhibit a plateau at the center of the wall where the magnetization is pointing perpendicu- lar to the wire and fitting of such a profile with the conventional tanh function leads to a significant underestimation of the real wall width. Rather we find from the analysis of the DW types that the opening angle $\alpha_{\text {DW }}$ of the triangular transverse walls, as shown in Fig. 1(b), constitutes a suitable quantity to characterize most DWs. After determining $\alpha_{\mathrm{DW}}$, an average DW width $w_{\text {DW }}$ can be calculated according to

$$
w_{\mathrm{DW}}=w_{c} \tan \left(\alpha_{\mathrm{DW}} / 2\right) \text {. }
$$

This is the physically relevant parameter, for instance, for electron transport across the wall. We observe an increase of $\alpha_{\mathrm{DW}}$ from $85^{\circ}$ for $w_{c, \mathrm{av}}=50 \mathrm{~nm}$ to $100^{\circ}$ for $w_{c, \mathrm{av}}=300 \mathrm{~nm}$ [Fig. 2(a)]. By calculating $w_{\text {DW }}$, we find an increase from 50 to $380 \mathrm{~nm}$ [Fig. 2(c)] averaged for all the walls in a certain range of $w_{c}$. No significant influence of the element width $w_{e}$ and the thickness of the material on $\alpha_{\mathrm{DW}}$, and therefore $w_{\mathrm{DW}}$, could be found in the range of thicknesses $(5-20 \mathrm{~nm})$ and $w_{e}(100-400 \mathrm{~nm})$ considered.

In order to simulate DWs in constrictions, we employed an extended classical Heisenberg model which can reproduce the changes in the spin structure at very short length scales as found in geometrically confined DWs. In this model, the magnetic moments are located on a cubic lattice with nearest neighbors having a ferromagnetic exchange coupling, a dipole-dipole interaction, and a coupling to an external magnetic field. ${ }^{22}$ The radius of curvature was kept constant to $1 \mu \mathrm{m}$ as in the experiments, and the element width $w_{e}$ was varied between 120 and $400 \mathrm{~nm}$ with a thickness of $4 \mathrm{~nm}$. The DW configurations for constriction widths $w_{c}$ in the

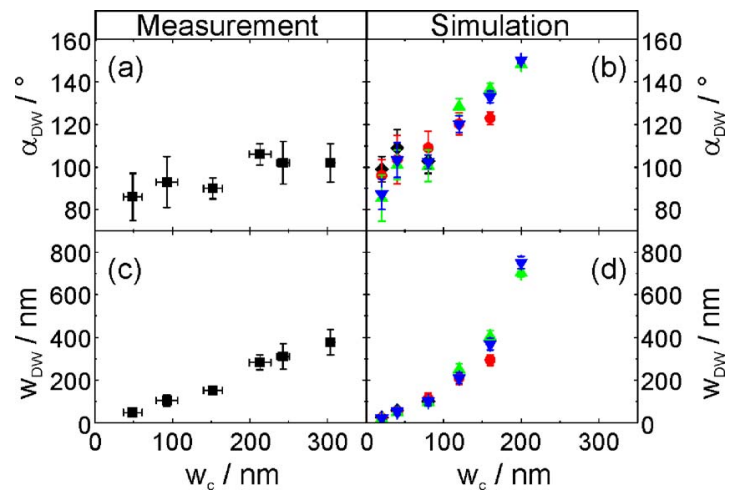

FIG. 2. (Color online) Dependence of DW angle $\alpha_{\mathrm{DW}}$ on an averaged constriction width $w_{c \text {,av }}$ obtained from (a) experiment and (b) Heisenberg simulations. [(c) and (d)] DW width $w_{\text {DW }}$ calculated using the DW angles $\alpha_{\text {DW }}$ from (a) and (b). In (b) and (d), the symbols refer to different element widths $w(\diamond=120 \mathrm{~nm}, \bigcirc=200 \mathrm{~nm}, \triangle=300 \mathrm{~nm}$, and $\nabla=400 \mathrm{~nm})$. 


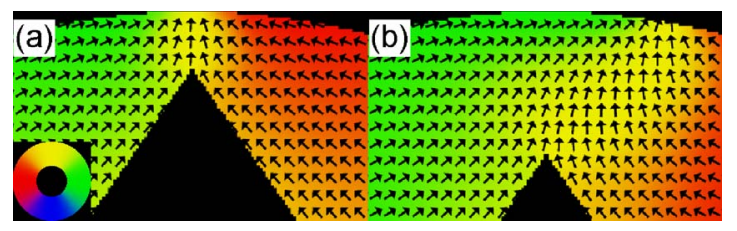

FIG. 3. (Color online) Magnetization configurations obtained from computer simulations of (a) symmetric and (b) asymmetric transverse DWs; shown are parts of the simulated structures with a thickness of $4 \mathrm{~nm}$ and constriction width $w_{c}$ /element width $w_{e}:$ (a) $80 \mathrm{~nm} / 400 \mathrm{~nm}$ and (b) $200 \mathrm{~nm} / 400 \mathrm{~nm}$. The color code in the inset of (a) and the arrows indicate the magnetization direction.

range of 20-200 nm were simulated. The notch had a triangular shape with a constant angle of $70^{\circ}$ in line with the experiment. The parameters of the Heisenberg simulations were deduced according to Ref. 21 from the material parameters of Permalloy; damping constant $\alpha=0.02$, exchange constant $A=13 \times 10^{-12} \mathrm{~J} / \mathrm{m}$, and saturation magnetization $M_{s}=800 \times 10^{3} \mathrm{~A} / \mathrm{m}$. We used 2 and $4 \mathrm{~nm}$ cell sizes with no significant difference in the results. In the experiment, DWs are formed after reducing an external magnetic field from saturation in the $y$ direction [see Fig. 1(a)] to zero. Since there is never a perfect alignment of the field to the constriction in the experiments, the field was tilted by $5.7^{\circ}$ to the $y$ axis in the simulation.

In the simulations, two types of transverse DWs were found within the constriction, as shown in Fig. 3: symmetric [Fig. 3(a)] and asymmetric [Fig. 3(b)] transverse DWs. The symmetric transverse DW is obtained in small constrictions, $w_{c}=20-80 \mathrm{~nm}$, and exhibits an elliptical shape, also observed in Ref. 14 . For wider constrictions, $w_{c} \geqslant 160 \mathrm{~nm}$, an asymmetric spin structure is favored with the direction of the DW tilt [to the right in Fig. 3(b)] governed by the initial field angle. For intermediate $w_{c}=120 \mathrm{~nm}$, we find both wall types depending on $w_{e}$. The key energy contributions to the DWs are the exchange energy, which favors large wall widths, and the stray field energy (shape anisotropy), which favors alignment of the spins parallel to the element edges. The increasing influence of the stray field energy results in smaller $w_{\text {DW }}$ for smaller constrictions. For symmetric walls, $w_{\mathrm{DW}}$ is comparable with the experimental values for $0<w_{c}<160 \mathrm{~nm}$ [Figs. 2(c) and 2(d)]. However, for $w_{c}>160 \mathrm{~nm}, w_{\text {DW }}$ extracted from the simulation increases to much larger values. The exact asymmetric DW tilt in experiment depends on irregularities such as the edge roughness, which are inherently not well known and thus not taken into account into the simulation. Together with the difficulty of determining the opening angle, this leads to the observed discrepancies of $w_{\text {DW }}$ for the highly asymmetric simulated walls with large $w_{c}$. The increase in the opening angle with $w_{c}$ means that $w_{\text {DW }}$, according to Eq. (1), increases more than linearly with increasing $w_{c}$. While this is clearly seen in Fig. 2(d), it is less obvious in Fig. 2(c) since the increase in the angle is smaller in the experiment than in the simulated data (as discussed above). Nevertheless we qualitatively see the same trend as in the experimental data.

In conclusion we have observed, depending on the geometry, asymmetric and symmetric transverse walls with electron holography, which is in agreement with micromagnetic predictions and confirmed by Heisenberg simulations.
In the measurements we found that, depending on the constriction width $w_{c}$, the asymmetric walls can be subdivided into tilted and buckled walls, the latter being an intermediate state just before the appearance of a vortex. We have also confirmed that the domain wall width $w_{\mathrm{DW}}$ depends strongly on $w_{c}$ and decreases with decreasing $w_{c}$. In agreement with simulations, the wall opening angle decreases with decreasing constriction width. This results in a faster than linear decrease of the wall width with $w_{c}$ which will facilitate the fabrication of very narrow domain walls where exciting physical effects are expected.

The authors acknowledge support by the Deutsche Forschungsgemeinschaft (SFB 513), by the Interreg III A program, and by the EPSRC (UK). One of the authors (C.S.) acknowledges support by the Landesstiftung BadenWürttemberg and thanks SSC, NIC, and the HLRS for computer time. Another author (R.E.D.-B.) acknowledges support by the Royal Society.

${ }^{1}$ D. A. Allwood, G. Xiong, C. C. Faulkner, D. Atkinson, D. Petit, and R. P. Cowburn, Science 309, 1688 (2005).

${ }^{2}$ S. S. P. Parkin, U.S. Patent No. 7031178 (2006).

${ }^{3}$ L. Berger, J. Appl. Phys. 55, 1954 (1984).

${ }^{4}$ A. Yamaguchi, T. Ono, S. Nasu, K. Miyake, K. Mibu, and T. Shinjo, Phys. Rev. Lett. 92, 077205 (2004).

${ }^{5}$ M. Kläui, P.-O. Jubert, R. Allenspach, A. Bischof, J. A. C. Bland, G. Faini, U. Rüdiger, C. A. F. Vaz, L. Vila, and C. Vouille, Phys. Rev. Lett. 95, 026601 (2005)

${ }^{6}$ Y. Nakatani, A. Thiaville, and J. Miltat, Nat. Mater. 2, 521 (2003).

${ }^{7}$ G. S. D. Beach, C. Nistor, C. Knutson, M. Tsoi, and J. L. Erskine, Nat. Mater. 4, 741 (2005).

${ }^{8}$ S. Zhang and Z. Li, Phys. Rev. Lett. 93, 127204 (2004).

${ }^{9}$ A. Thiaville, Y. Nakatani, J. Miltat, and Y. Suzuki, Europhys. Lett. 69, 990 (2005).

${ }^{10}$ J. Xiao, A. Zangwill, and M. D. Stiles, Phys. Rev. B 73, 054428 (2006).

${ }^{11}$ P. M. Levy and S. Zhang, Phys. Rev. Lett. 79, 5110 (1997).

${ }^{12}$ A. D. Kent, J. Yu, U. Rüdiger, and S. S. P. Parkin, J. Phys.: Condens. Matter 13, R461 (2001).

${ }^{13}$ P. Bruno, Phys. Rev. Lett. 83, 2425 (1999).

${ }^{14}$ R. D. McMichael and M. J. Donahue, IEEE Trans. Magn. 33, 4167 (1997).

${ }^{15}$ M. Laufenberg, D. Backes, W. Bührer, D. Bedau, M. Kläui, U. Rüdiger, C. A. F. Vaz, J. A. C. Bland, L. J. Heyderman, F. Nolting, S. Cherifi, A. Locatelli, R. Belkhou, S. Heun, and E. Bauer, Appl. Phys. Lett. 88, 052507 (2006).

${ }^{16}$ Y. Nakatani, A. Thiaville, and J. Miltat, J. Magn. Magn. Mater. 290, 750 (2005).

${ }^{17}$ P.-O. Jubert, R. Allenspach, and A. Bischof, Phys. Rev. B 69, 220410(R) (2004).

${ }^{18}$ C. Brownlie, S. McVitie, J. N. Chapman, and C. D. W. Wilkinson, J. Appl. Phys. 100, 033902 (2006).

${ }^{19}$ M. Kläui, H. Ehrke, U. Rüdiger, T. Kasama, R. E. Dunin-Borkowski, D. Backes, L. J. Heyderman, C. A. F. Vaz, J. A. C. Bland, G. Faini, E. Cambril, and W. Wernsdorfer, Appl. Phys. Lett. 87, 102509 (2005).

${ }^{20} \mathrm{H}$. Kronmüller and M. Fähnle, Micromagnetism and the Microstructure of Ferromagnetic Solids (Cambridge University Press, Cambridge, 2003).

${ }^{21} \mathrm{~A}$. Aharoni, Introduction to the Theory of Ferromagnetism (Oxford University Press, Oxford, 2001).

${ }^{22}$ C. Schieback, M. Kläui, U. Nowak, U. Rüdiger, and P. Nielaba, online available at Eur. Phys. J. B (2007), DOI:10.1140/epjb/e2007-00062-2 www.edpsciences.org/articles/epjb/abs/first/b06640/b06640.html.

${ }^{23}$ D. Backes, L. J. Heyderman, C. David, R. Schäublin, M. Kläui, H. Ehrke, U. Rüdiger, C. A. F. Vaz, J. A. C. Bland, T. Kasama, and R. E. DuninBorkowski, Microelectron. Eng. 83, 1726 (2006).

${ }^{24}$ R. E. Dunin-Borkowski, M. R. McCartney, B. Kardynal, S. S. P. Parkin, M. R. Scheinfein, and D. J. Smith, J. Microsc. 200, 187 (2000).

${ }^{25}$ A. Hubert and R. Schäfer, Magnetic Domains: The Analysis of Magnetic Microstructures (Springer, Berlin, 1998). 